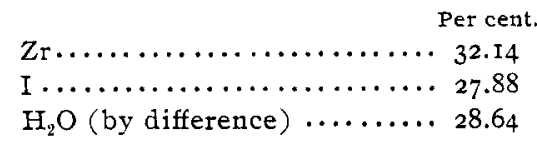

It lost only II.O7 per cent. of its weight after three hours' heating at $100^{\circ}-120^{\circ}$.

UNIVFRSITY OF NORTH CAROLINA.

\title{
ATROPINE PERIODIDES AND IODOMERCURATES.
}

By H. M. Gordin ANd A. B. PREscott.1

Received March 12, 1898.

\section{ATROPINE ENNEAIODIDE.}

$T$ has been known for a long time that in solutions of atropine salts, as in those of the salts of most other alkaloids, a solution of iodine in potassium iodide gives an insoluble precipitate. The nature of this precipitate has so far as we know not been thoroughly investigated. Jörgensen ${ }^{2}$ has obtained and described two periodides of atropine : a triiodide and a pentaiodide Our experience has taught us that in aqueous solutions the capacity of atropine for combining with iodine varies very widely, and the quantities taken up seem to depend upon the concentration of the liquids and even the order of mixing them.

The highest number of iodine atoms which a molecule of atropine can combine with, seems to be nine; between this and the above-mentioned triiodide lie the other compounds of atropine with iodine which are formed in aqueous solution. The same is true when chloroform is used as a solvent. We reserve for a later date a report upon the exact conditions which are necessary for the formation of any particular periodide of atropine. For the present we wish to say that under the conditions described below we were able to obtain the enneaiodide of atropine, $\mathrm{C}_{17} \mathrm{H}_{23} \mathrm{NO}_{3}$. HI. $\mathrm{I}_{8}$, as the sole combination of all the alkaloid, and sufficiently stable.

These conditions are the following: The concentration of the aqueous solution of the atropine salt should not exceed fivetenths per cent.; that of the iodine solution must not exceed one

1. In the work of Research Committee D, Section 2, Committee on Revision of the Pharmacopeia of the United States.

2 J.prakt. Chem. [2], 3, 329. 
per cent.; the latter has to be acidulated with some sulphuric or hydrochloric acid; the atropine solution must be added to the iodine solution and not vice versa, and this addition must take place in small portions at a time, shaking the mixture thoroughly after each addition. At first the liquid becomes very turbid and particles of iodine are seen to be floating upon its surface : on continued addition of the atropine solution and shaking, the iodine-colored turbidity disappears, a dark granular precipitate falls out, and the supernatant liquid becomes perfectly transparent but is still dark-colored. If the addition of the atropine be stopped at this stage, $i . e$, while the supernatant liquid has a very dark red color, the composition of the precipitate will be found to be that of the enneaiodide $\mathrm{C}_{17} \mathrm{H}_{23} \mathrm{NO}_{3} \mathrm{HI}$. I.

Whether additional quantities of atropine will make the precipitate take up more atropine and become a lower periodide we shall try to determine by later experiments. On the other hand when the order is reversed and the iodine solution is added to the atropine solution, it is always a lower periodide that is formed; but whether on continued addition of the iodine the precipitate will take up more of it and become a higher periodide, we cannot say as yet.

The enneaiodide, obtained as described, being unstable while moist and when removed from its mother-liquor, the precipitate has to be collected quickly by means of a pump, washed a few times with cold water and dried first on porous plates and then in vacuum over sulphuric acid. As thus obtained it is a very dark brown almost black powder, quite permanent in dry air and has only a slight odor of iodine. It is very difficultly soluble in ether, chloroform, benzene, or carbon disulphide, but is soluble in alcohol, very freely when hot. In cold water it is insoluble; hot water decomposes it quickly; it is also decomposed by concentrated solutions of potassium iodide. At $90^{\circ} \mathrm{C}$. it commences to give up iodine vapors and at $140^{\circ} \mathrm{C}$. melts to a dark liquid.

To obtain it in crystalline form it is first washed with a little cold alcohol to remove traces of free iodine and then dissolved in warm alcohol. On cooling it crystallizes out in dark-green prisms and leaflets, having the same properties as the noncrystallized body. 
In this enneaiodide one-ninth of the total iodine is firmly combined just as in normal hydriodides, while eight-ninths is easily removed by reducing agents, such as sulphur dioxide and sodium thiosulphate. The compound therefore may be considered an atropine hydriodide octaiodide.

The additive iodine we estimated volumetrically and the total iodine both gravimetrically and volumetrically.

To estimate the additive iodine a small quantity of the enneaiodide is dissolved in very little alcohol, an excess of a standardized solution of sodium thiosulphate added and the excess titrated back with a standard solution of iodine using starch as the indicator.

For total iodine the substance is covered with an excess of powdered metallic zinc and some water and then boiled gently for ten or fifteen minutes, taking care to prevent loss by spurting; the mixture is then thrown upon a filter, and the containing flask and the filter thoroughly washed with hot water. The iodine in the zinc iodide thus formed can either be estimated by precipitation with silver nitrate and nitric acid and weighing as silver iodide, or it is precipitated with an excess of a standard. ized solution of silver nitrate and the excess titrated back with a standard solution of ammonium thiocyanate, using ferric nitrate as indicator.

Having obtained the enneaiodide of atropine it was natural to suppose the existence of a heptaiodide, with probability of a complete series from the triiodide to the enneaiodide. And in fact in the course of our work we once obtained this heptaiodide. But our efforts to determine the exact conditions necessary for the formation of this body have so far not been successful.

The easiest way to obtain the periodides of atropine is to use chloroform as a solvent. On adding twenty grams atropine to a warm solution of thirty grams iodine in chloroform (500 cc.) the enneaiodide crystallizes out very soon in the shape of small, shining, dark green crystals. If these be removed by filtration, the mother-liquor will give several successive crops of the dark blue pentaiodide and at last a crop of the brownish-red triiodide.

II. ATROPINE MERCURIC IODIDES.

The periodides of atropine, like those of many other alkaloids, 
easily give double salts with mercuric iodide, obtained on shaking the alcoholic solution of the periodide with mercury and gently warming the mixture. This double iodide of atropine and mercury can also be made by mixing theoretical quantities of atropine and iodine, adding a little alcohol and an excess of mercury, slightly warming, and shaking till the color of iodine disappears. If the higher periodides of atropine be used in the preparation of this double iodide of atropine and mercury there is always separation of mercurous iodide; but if theoretical quantities of atropine and iodide are used there is no separation of mercurous iodide. The formula of this double iodide of mercury and atropine, as shown by our analysis, seems to be $\mathrm{C}_{12} \mathrm{H}_{23} \mathrm{NO}_{3} \cdot \mathrm{HI} \cdot \mathrm{HgI}_{2}$. It resembles in composition several other double iodides of alkaloids and mercury obtained by Groves by a different method. But besides this double iodide we also obtained another having the composition (Atrop.HI) ${ }_{2} . \mathrm{HgI}_{2}$. It was made by treating a solution of the Atrop.HI. HgI in diluted alcohol with an excess of potassium iodide. From the liquid obtained by shaking an alcoholic solution of atropine and iodine with mercury the monoatropine hydriodide mercuric iodide crystallizes out in shining yellow crystals, melting at $89^{\circ}$ to $90^{\circ} \mathrm{C}$, difficultly soluble in ether or chloroform, partly soluble in hot water, and very soluble in warm alcohol. The diatropine hydriodide mercuric iodide obtained as said above by the action of potassium iodide on the mono compound, crystallizes in perfectly white, silky needles, which on being dried assume a yellowish tint. They melt at $98^{\circ}$ to $99^{\circ} \mathrm{C}$., are very easily soluble in warm alcohol, and quite soluble in hot water.

The analysis for mercury and for iodine in these bodies can be made in different ways, but the best results are obtained by a modification of the method of Risse." The mercury and the iodine are determined in two separate portions. For mercury the substance is dissolved in a little warm alcohol and a little water added; the solution is then acidulated with a few drops of dilute hydrochloric acid and saturated with hydrogen sulphide. The mercuric sulphide is then dried and weighed in the usual way. For the determination of iodine the substance is treated

1 Quart.J. of Chem. Soc., 11, 97.

2 Ann. Chenn. (Liebig), 107, 223. 
with a hot solution of potassium hydroxide ( $I:$ IO), diluted with water, filtered, and when completely cold neutralized with acetic acid. The iorline is now precipitated with silver nitrate and nitric acid and determined as silver iodide in the usual way.

In the following analyses the standard silver nitrate solution was made to correspond with one per cent. of iodine and the ammonium thiocyanate solution to correspond, cc. per cc., with the silver nitrate solution. The standard solution of iodine contained one per cent. of iodine and the sodium thiosulphate solution corresponded, cc. per cc., with the iodine solution.

Analysis of the Atropine Enneaiodide not Recrystallized Volumetric estimation for total iodine, 0.15225 gram required i 2.2 cc. of the silver nitrate solution.

For the iodine removed by reduction, 0.19255 gram required I $3.7 \mathrm{cc}$. of the thiosulphate solution.

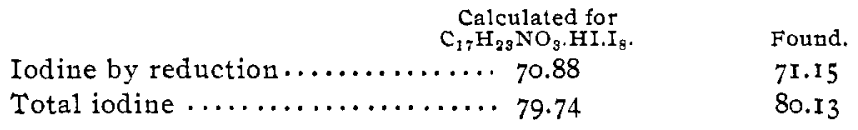

In the gravimetric estimation from 0.2 I 8 was obtained 0.3207 silver iodide.

$$
\begin{aligned}
& \begin{array}{l}
\text { Calculated for } \\
\mathrm{C}_{17} \mathrm{H}_{29} \mathrm{NO}_{9} \cdot \mathrm{HII} \mathrm{I}_{8}
\end{array} \quad \text { Found. } \\
& \text { Total iodine } . \ldots \ldots \ldots \ldots \ldots \ldots \ldots .79 .74 \quad 79.48
\end{aligned}
$$

Analysis of the Atropine Enneaiodide Recrystallized from Alcohol: The methods employed were the same as with the nonrecrystallized compound and the standard solutions of the same strength.

In the volumetric estimation of total iodine, 0.12685 gram required IO.I cc, of the silver nitrate solution. For the iodine removed by reducing agents, 0.23105 gram required $\mathrm{I} 6.4 \mathrm{cc}$. of the thiosulphate solution.

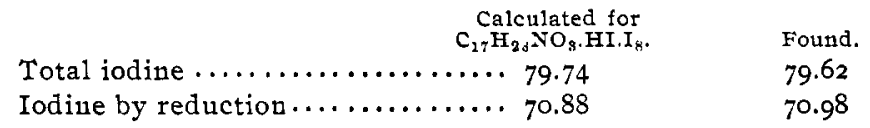

In the gravimetric estimation of total iodine, $0.203 \mathrm{I}$ gram

\begin{tabular}{|c|c|}
\hline & $\begin{array}{c}\text { Calculated for } \\
\mathrm{C}_{17} \mathrm{H}_{29} \mathrm{NO}_{3} . \mathrm{MI} \cdot \mathrm{I}_{8}\end{array}$ \\
\hline Total iodine & … 79.74 \\
\hline
\end{tabular}
gave 0.30035 silver iodide. 
Analyses of the Double Salts of Atropine Hydriodide and Mercuric Iodide: 0.2532 gram of the monoatropine hydriodide mercuric iodide gave 0.0699 gram mercuric sulphide, and 0.30445 gram of the substance gave 0.24785 gram silver iodide.

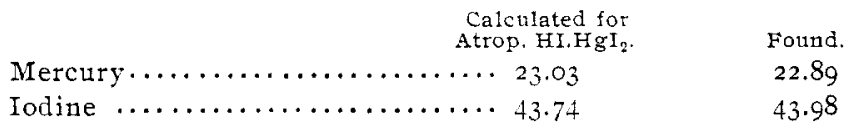

Of the diatropinehydriodide mercuric iodide, 0.1798 gram gave 0.032 gram mercuric sulphide, and 0.257 gram of the substance gave 0.18885 gram silver iodide.

\begin{tabular}{|c|c|c|}
\hline & $\begin{array}{l}\text { Calculated for } \\
\text { (Atrop.HI) } \text { Ag }_{2} \cdot \mathrm{Hg}_{2}\end{array}$ & Found. \\
\hline Mercury & $\ldots \ldots{ }^{\prime} 5.5^{8}$ & $I 5 \cdot 34$ \\
\hline Iodine... & $\ldots .39 .46$ & 39.70 \\
\hline
\end{tabular}

III. ATROPINE ESTIMATION AS PERIODIDE.

The formation of atropine enneaiodide under the conditions described above affords a convenient method for estimating the strength of atropine solutions by means of a standard solution of iodine and titrating back with a standard solution of sodium thiosulphate. The way to operate is as follows: The atropine solution, whose strength is approximately determined by a preliminary experiment, operating in the way described below, is diluted with water so as to contain at most four-tenths to fivetenths per cent. of the alkaloid and in so doing the solution is made up to a given volume. Twenty or thirty $\mathrm{cc}$. of a decinormal iodine solution are now put into a measuring flask holding Ioo cc., diluted with a little water, and acidulated with a few drops of diluted sulphuric acid; the atropine solution is now run in from a burette slowly, and in small portions at a time, shaking the flask thoroughly after each addition and observing whether the supernatant liquid conmences to clear up. If not, a little more of the atropine solution is added, and the mixtures again shaken for a minute or two. In this way the addition of atropine is slowly continued till after a few shakings the supernatant liquid commences to become transparent, still having a dark red color, which can easily be seen by stoppering the flask, reversing it and looking through its neck. The addition of atropine is then stopped, the flask shaken till the supernatant liquid 
is perfectly transparent, and then it is diluted to Ioo cc. Of this liquid an aliquot portion is filtered off and the excess of iodine in it determined by a decinormal solution of sodium thiosulphate. Every part by weight of iodine consumed corresponds to 0.2849 part of alkaloid or every cubic centimeter of the decinormal iodine solution consumed corresponds to 0.0036048 gram of atropine alkaloid.

These factors are obtained on the ground that for the formation of the atropine enneaiodide only eight atoms of iodine are supplied by the free iodine used to make the decinormal iodine solution, the ninth atom coming from the potassium iodide of that solution. The success of this method depends solely upon the atropine solution being sufficiently diluted, as otherwise a resinous mass is liable to be formed instead of a granular precipitate, and added in small portions at a time to the iodine solution, care being taken to shake the nixture after each addition and to stop the operation as soon as the supernatant liquid commences to become transparent, being still red-colored. It is easy to be guided by the very dark color, as an excess of atropine makes the liquid almost colorless. With a little care the method gives very good results, as can be seen from the following analyses.

Whether this method of assay is applicable to the crude drugs containing atropine and their galenical preparations, we shall try to determine by experiments to be continued.

In conclusion we wish to say that the method of using a solution of iodine and potassium iodide for the quantitative estimation of alkaloids has been long since proposed, ${ }^{1}$ and recently Kippenberger ${ }^{2}$ has given considerable prominence to this method in his toxicological and other researches. But he rests upon the assumption of the formation of the atropine triodide, and this may be the leading product under the particular conditions he prescribes ; but his method as a whole, when tried in this laboratory, has not given satisfactory results. The estimation of caffein as a periodide recently published by Gomberg ${ }^{3}$ proves highly satisfactory.

\footnotetext{
1 Bouchard : Compt. rend., 9, 475; R. Wagner: Ding. poly. J., 16r, 40; Ztschr, anal. Chem., I, I02.

2 Ztschr. anal. Chem, 35, Io; 34, 3I7, etc.

8 This Journal, $18,33^{\mathrm{I}}$.
} 
In the following estimation of the strength of atropine solutions both the iodine solution and the sodium thiosulphate solution were strictly decinormal. Of the iodine solution twenty or twenty-five $c c$, were put into a Ioo-cc. measuring flask and atropine solutions of known strength run in from a burette and the operation continued as described above. When the supernatant liquid was perfectly transparent the flask was filled up to roo cc. and fifty cc. were filtered off. In these fifty cc. the excess of iodine was determined by means of the tenth-normal thiosulphate solution and the strength of the atropine solutions calculated by using the factor 0.0036048 for every cubic centimeter of decinormal iodine used up.

\begin{tabular}{|c|c|c|c|c|}
\hline No. of sample. & $\begin{array}{l}\text { Number of cc. } \\
\text { of the atropine } \\
\text { solutiou takets. }\end{array}$ & $\begin{array}{l}\text { Number of cc. of } \\
\text { the tenth-normal } \\
\text { iodine used up. }\end{array}$ & $\begin{array}{l}\text { Calculated } \\
\text { strength. } \\
\text { Per cent. }\end{array}$ & $\begin{array}{l}\text { Actual } \\
\text { strength. } \\
\text { Per cent. }\end{array}$ \\
\hline I & I2 & I 5.6 & 0.47 & 0.50 \\
\hline 2 & I5 & I 4.9 & 0.36 & 0.40 \\
\hline 3 & I7 & $\mathrm{I}_{3} .2$ & 0.28 & 0.30 \\
\hline 4 & I2 & 10.0 & 0.28 & 0.25 \\
\hline
\end{tabular}

We shall try to determine experimentally whether this method is also applicable to other alkaloids and their galenical forms.

The above-mentioned factor for every cubic centimeter of the decinormal iodine solution was deduced as we said upon the ground that only eight atoms of iodine of every molecule of the atropine enneaiodide are furnished by the free iodine of the iodine solution-the ninth atom forming the normal iodine of the enneaiodide coming from the potassium iodide.

That this is really so has been admitted by almost all chemists in the case of all other periodides whose constitution is similar to that of the atropine enneaiodide. It would, therefore, seem to be superfluous to prove experimentally the correctness of this view with regard to the enneaiodide of atropine. But owing to the fact that Kippenberger in the above-cited articles advances a different view we thought it advisable to prove irrefutably that the iodide of potassium of the iodide solution takes an active part in the reaction by which the atropine enneaiodide is formed and gives off an amount of iodine which is just equal to the amount of the normal iodine of the enneaiodide.

We proceeded in the following manner: We first determined the strength of our iodine solution with regard to free iodine; 
this was done by means of a sodium thiosulphate solution of known strength. We then took ten $\mathrm{cc}$. of this iodine solution and determined the total amount of iodine, free as well as bound to potassium, by shaking them in a fiask with an excess of powdered metallic zinc till the color of iodine disappeared, filtering the solution of zinc iodide and potassium iodide thus obtained, washing the flask and the excess of zinc with hot water and precipitating the iodides in the filtrate with silver nitrate and nitric acid. From the weight of silver iodide, washed and dried in the usual way, we calculated the amount of total iodine present in our iodine solution. By subtracting the amount of free iodine from that of the total iodine we obtained the amount of iodine present in our solution as potassium iodide. We thus found that the iodine solution contained one per cent. free iodine and r.23r 2 per cent. combined iodine.

We then put forty $\mathrm{cc}$. of this iodine solution in a IOO-cc. measuring lask, added twenty cc. of a three-tenths per cent. solution of atropine alkaloid, and, after shaking well, diluted the liquid in the flask to $\mathrm{I} O \mathrm{cc}$. We now took two vials and in each filtered off twenty-five $c c$, from the flask. In the first vial the amount of free iodine titrated back with sodium thiosulphate was found to be 0.0465 . From this it is easy to deduce that the twenty $c c$. of the three-tenths per cent. atropine solution consumed 0.214 gram of the free iodine. In the second vial the total iodine was determined in the same way as described above and the quantity of silver iodide was found to be $0.3023 \mathrm{gram}$ which shows that after the treatment with atropine the liquid contained 1.633 per cent. total iodine. From these data we deduce that the twenty $\mathrm{cc}$. of the atropine solution have taken up 0.02528 gram iodine from the bound iodine. As the twenty cc. of atropine solution contained 0.06 gram atropine we find for 100 parts of precipitated enneaiodide

\begin{tabular}{|c|c|c|}
\hline & $\begin{array}{l}\text { Found. } \\
\text { Per cent. }\end{array}$ & $\begin{array}{l}\text { Calculated. } \\
\text { Per cent. }\end{array}$ \\
\hline Atropine $\ldots \ldots$. $\ldots \ldots \ldots \ldots \ldots \ldots$ & 20.05 & 20.26 \\
\hline $\begin{array}{l}\text { Iodine taken from the free iodine of } \\
\text { the iodine solution.............. }\end{array}$ & $7 \mathrm{I} \cdot 5^{\circ}$ & 70.88 \\
\hline Iodine from the iodine bound as KI.. & 8.45 & 8.86 \\
\hline
\end{tabular}

We see that in aqueous acidulous solutions the potassium iodide takes an active part in the reaction by joining in a yield of hy- 
driodic acid for the normal hydriodide of the alkaloid, necessary to hold the additive iodine of the periodide. When carbon disulphide or carbon tetrachloride is used as solvent for the atropine and the iodine, no periodide seems to be formed, possibly for the reason of there being no generation of hydriodic acid for the making of hydriodide. As to the formation of the periodides in chloroformic solutions of atropine and iodine, it might possibly be explained by the substituting action of iodine either upon the chloroform or upon a side-chain of the atropine.

The direct substitution of iodine for some of the hydrogen in these bodies might, in this case, be made possible by the presence of the natural base which combines with the hydriodic acid, when formed by such a substitution. Part of the atropine would then act in a manner similar to that of mercuric oxide, which is usually employed in the iodizing of hydrocarbons. An analogous case we have in the easy substitution of iodine for some of the hydrogen in aniline, a part of which combines with the hydriodic acid, which is set free through the substitution of iodine for hydrogen in another part. At any rate we shall endeavor to determine the by-products formed when atropine enneaiodide is produced in chloroformic solutions.

UNIVERSITY OF MICHIGAN,

FEBRUARY 28 , Isgs.

\section{COMPOSITION OF THE ASHES OF SOME RAW TANNING MATERIALS.'}

BY WM. K. ALsop aND J. H, Yoctm. Received March $15, \pm 898$.

NO systematic investigation of tannin sources has been pubthe other chemical compounds found in the raw material. With the object of making a complete analysis of some of the common sources of tannin, we present this paper on the ash and tannin analysis, and hope in the future to present others bearing upon the sugars and other soluble non-tannins, celluloses, and red coloring material.

In the table presented showing the tannin content of oak and hemlock bark, the averages from two widely separated localities for each of a large number of analyses, are given. The method

1 Read before the New York Section, March II, 1898. 